\title{
Two amino acids in glutamic acid decarboxylase act in concert for maintainance of conformational determinants recognised by Type I diabetic autoantibodies
}

\author{
T. I.M. Tree ${ }^{1}$, N. G. Morgenthaler ${ }^{1}$, N. Duhindan ${ }^{1}$, K. E. Hicks ${ }^{1}$, A.-M. Madec ${ }^{2}$, W. A. Scherbaum ${ }^{3}$, J.P. Banga ${ }^{1}$ \\ ${ }^{1}$ Guy's, King's, St Thomas' School of Medicine, Division of Medicine, London, UK \\ 2 INSERM 449, Faculty of Medicine RTH Laennec, Lyons, France \\ ${ }^{3}$ Diabetes Research Institute, University of Düsseldorf, Düsseldorf, Germany
}

\section{Abstract}

Aims/hypothesis. Glutamic acid decarboxylase 65 is a major autoantigen in Type I (insulin-dependent) diabetes mellitus, autoimmune polyendocrine syndrome and stiff-man syndrome. These disorders are characterised by the presence of multiple autoantibodies to the autoantigen which can be distinguished in a variety of different ways. We have investigated the role of single amino-acid mutations in glutamic acid decarboxylase 65 in distinguishing the binding of serum antibodies and a variety of patient-derived human IgG monoclonal antibodies directed to different determinants of the autoantigen.

Methods. We identified a mutant of glutamic acid decarboxylase 65 that contained four single amino-acid mutations from the wild-type molecule. The role of these mutations was investigated by site-directed mutagenesis. We investigated the binding of patientderived serum antibodies to glutamic acid decarboxylase 65 to a number of single and double amino-acid mutants using immunoprecipitation with labelled, recombinant antigen. To overcome the heterogeneity of different anti-glutamic acid decarboxylase 65 antibodies present in a patient's serum, the binding of a panel of eleven patient-derived human monoclonal antibodies recognising different determinants on the autoantigen was also studied.

Results. Two replacements in glutamic acid decarboxylase 65 at Asn247Ser and Leu574Pro were identified that preferentially influence the anti-glutamic acid decarboxylase 65 serum antibodies of Type I diabetic patients, without statistically significantly effecting those recognised in other disorders. Single or double amino-acid replacements Asn247Ser and Leu574Pro in the autoantigen showed differential affects on expression of epitopes recognised by the human monoclonals. The double replacement of Asn247Ser and Leu574Pro in glutamic acid decarboxylase 65 resulted in the loss of binding of all eleven human monoclonal antibodies, irrespective of their epitope recognition. In contrast, single replacement of Leu574Pro statistically significantly reduced the binding of some carboxyl terminal-directed antibodies such as MICA 1, MICA 3 and DP-A without influencing the binding of other monoclonals. Replacement of Asn247Ser did not, however, influence the binding of any patients serum or human monoclonal antibodies.

Conclusion/interpretation. Two distantly spaced amino acids, Asn247 and Leu574 in glutamic acid decarboxylase 65 were identified that act in concert to greatly influence the conformational structure of the autoantigen and statistically significantly influence the binding of antibodies present in Type I diabetic sera. The single or double amino-acid mutants can be used to distinguish some anti-glutamic acid decarboxylase- 65 autoantibodies and could prove useful in distinguishing Type I diabetic from autoimmune polyendocrine syndrome and stiff-man syndrome patients' sera as well as to study changes in antibody patterns during disease progression. [Diabetologia (2000) 43: 881-889]

Keywords Glutamic acid decarboxylase 65, autoimmune polyendocrine syndrome, stiff-man syndrome, human monoclonal antibodies, mutants.
Received: 9 December 1999 and in revised form: 29 February 2000

Corresponding author: Dr J.P. Banga, GKT School of Medicine, Division of Medicine, Bessemer Road Campus, Bessemer Road, London, SE5 9PJ, UK
Abbreviations: APS, Autoimmune polyendocrine syndrome; hmABs, human monoclonal antibodies; mutation A, Trp9Arg; mutation B, Asn247Ser; mutation C, Tyr425Asn; mutation D, Leu574Pro; SMS, stiff-man syndrome. 
The enzyme glutamic acid decarboxylase (GAD) is responsible for the synthesis of the major inhibitory neurotransmitter, $\gamma$-aminobutyric acid (GABA) [1]. Two related isomers $\mathrm{GAD}_{65}$ and $\mathrm{GAD}_{67}$, encoded by non-allelic genes and sharing an identical exon-intron structure have been described [2-4]. The GAD isomers are expressed predominantly in the GABA-ergic neurons of the central nervous system and in pancreatic islet beta cells, although there are species differences in their expression in the islet beta cells [1, 5]. At the protein level, $\mathrm{GAD}_{65}$ (of 585 residues) and $\mathrm{GAD}_{67}$ (of 594) residues are highly dissimilar in the amino terminal 100 residues with a $25 \%$ homology whereas the remainder of the molecule shows around $75 \%$ homology $[2,3]$.

Glutamic acid decarboxylase is also a major target in autoimmunity, with autoantibodies being present in new-onset Type I (insulin-dependent) diabetes mellitus, autoimmune polyendocrine syndrome (APS) and stiff-man syndrome (SMS) patients, where they serve as important markers of disease activity [6-14]. Autoantibodies to GAD in new-onset Type I diabetic patients are specifically directed to the $\mathrm{GAD}_{65}$; a small proportion of antibodies to the larger isoform $\mathrm{GAD}_{67}$ are also present but these can be blocked with $\mathrm{GAD}_{65}$ indicating there are shared determinants between the molecules $[15,16]$. Autoantibodies to $\mathrm{GAD}_{65}$ are present in $70-80 \%$ of new-onset Type I diabetic patients but their titre is considerably lower than those in APS and SMS patients [17-20]. There are also other differences between the anti$\mathrm{GAD}_{65}$ antibodies present in the sera from new-onset Type I diabetic patients and that from patients with APS and SMS. For example, anti-GAD ${ }_{65}$ antibodies from Type I diabetic patients generally do not react with linear peptide sequences or with denatured antigen and thus are dependent on binding to discontinous determinants brought together in the three-dimensional folding of the protein [6,18-24]. Furthermore, antibodies present in APS and SMS sera inhibit the enzymatic activity of $\mathrm{GAD}_{65}$ but the antibodies in Type I diabetic sera do not show this property [25, 26].

The determinants recognised by the anti-GAD 65 antibodies present in new-onset Type I diabetic patients have been delineated using chimeras of $\mathrm{GAD}_{65}$ and $\mathrm{GAD}_{67}[16,21,22]$. The antibodies target epitopes in the middle region (residues 240 to 435) and the carboxyl terminal region (residues 451 to 570) of $\mathrm{GAD}_{65}$, termed IDDM-E1 and IDDM-E2, respectively $[21,22]$. These and other studies using human monoclonal antibodies (hmABs) to $\mathrm{GAD}_{65}$ from patients indicate that the epitope recognition of $\mathrm{GAD}_{65}$ in new-onset Type I diabetic patients is diverse, with recognition of multiple determinants on the molecule [27-32]. Although the structure of GAD is not known, in a recent study a model was built of the middle (residues 200 to 460 ) and the carboxyl region (residues 461 to 585) of $\mathrm{GAD}_{65}$ based on the three-dimensional structures of related decarboxylases and the autoreactive determinants recognised by the hmABs were fine mapped[33]. The model shows that the middle and carboxyl regions form two very separate folding domains where virtually the entire surface of $\mathrm{GAD}_{65}$ is targeted by the autoimmune response [33]. In this study we report on single amino-acid mutagenesis analysis of $\mathrm{GAD}_{65}$ to define the determinants recogised by hmABs to $\mathrm{GAD}_{65}$. The results identify two amino-acid replacements in $\mathrm{GAD}_{65}$ that seem to act together in a concerted manner to ablate the binding of a variety of hmABs directed to different determinants of the molecule.

\section{Materials and methods}

Patients' sera and human IgG monoclonal antibodies to $G A D_{65}$. Sera from new-onset Type I diabetic, APS and SMS patients were obtained after informed consent of the patients and ethical approval from the institution was given. Sera were obtained from new-onset Type I diabetic patients when the patients were first seen in the clinic or within 4 weeks of clinical diagnosis. Patients with APS were diagnosed on the criteria of islet-cell antibody positivity, absence of diabetes and documented presence of two other organ-specific autoantibodies [34]. Stiff-man syndrome was diagnosed according to clinical symptoms and none of these patients had diabetes. Only GAD antibody-positive sera (assay validated in the 2nd International GAD antibody workshop with a sensitivity of $87 \%$ and a specificity of $91 \%$ ) [35] were included in these studies. A total of eleven hmABs to $\mathrm{GAD}_{65}$ were used for immunoprecipitation; these comprised MICA 1, MICA 2, MICA 3, MICA 4, MICA 6, DP A, DP B, DP C, DP D, b78 and b96 [27, 29, 31] and were used as antibody-containing tissue culture supernatants. The b96 antibody used in this study was derived from the $\mathrm{B}$ cell line and recognises the C-terminal region of $\mathrm{GAD}_{65}$ $[31,32]$; subsequent cloning of the cell line produced the b96.11 clone which recognises the middle region of $\mathrm{GAD}_{65}$ with similar epitope recognition to MICA 4 [33] but this clone was not used in this study.

Cloning of GAD from human islet cells. Total RNA was prepared from 10000 pelleted and purified human islet cells (from Professor RG Bretzel, Giessen, Germany). We did PCR for $\mathrm{GAD}_{65}$ cDNA with the primers 5 'TCTCCGGATCCGATG GCATCTCCGGCTCT3' (BQ0) and 5'GAGCAGAATTCTTATAAATCTTGTCCAAGGC3' (BQ1) (containing EcoRI and a Bam HI sites, respectively) using $2.5 \mathrm{U} \mathrm{Taq}$ polymerase (Bioline, London, UK) for 30 cycles $\left(40 \mathrm{~s}\right.$ at $92^{\circ} \mathrm{C}, 90 \mathrm{~s}$ for $55^{\circ} \mathrm{C}$ and $240 \mathrm{~s}$ at $\left.72^{\circ} \mathrm{C}\right)$. The faint band at $1.8 \mathrm{~kb}$ was purified and re-amplified as above using $\mathrm{BQ} 0 / \mathrm{BQ} 1$ primers and two internal primers flanking the unique Nar1 site in $\mathrm{GAD}_{65}$ (primer A, 5 'GGCAGACATGGCTGACATCAAC3' and primer B, 5 'TTCTGTTTGGCTTCAAG AAT3') and subcloned into Bluescript (pBS) (Stratagene, Cambridge, UK). Sequencing of the full-length $\mathrm{GAD}_{65} \mathrm{cDNA}$ showed that the clone contained eight single base-pair changes from the wild-type sequence (Table 1). Of the base-pair alterations four were silent mutations; the other base-pair changes resulted in the following changes; Trp9Arg, Asn247Ser, Tyr425Asn and Leu574Pro (Table 1). We cloned $\mathrm{GAD}_{67}$ cDNA as described [31]. 
Table 1. Base-pair alterations in the mutant $\mathrm{GAD}_{65}$ from the sequence of wild-type molecule

bp25, $\mathrm{T} \rightarrow \mathrm{C}$; amino acid (change) Trp $\rightarrow$ Arg

bp594, A $\rightarrow$ C; amino acid (silent) Ser $\rightarrow$ Ser

bp740, A $\rightarrow$ G; amino acid (change) Asn $\rightarrow$ Ser

bp1273, $\mathrm{T} \rightarrow \mathrm{A}$; amino acid (change) Tyr $\rightarrow$ Asn

bp1410, $\mathrm{T} \rightarrow \mathrm{C}$; amino acid (silent) His $\rightarrow$ His

bp1434, A $\rightarrow$ G; amino acid (silent) Ala $\rightarrow$ Ala

bp1515, C $\rightarrow$ T; amino acid (silent) Cys $\rightarrow$ Cys

bp1721, T $\rightarrow$ C; amino acid (change) Leu $\rightarrow$ Pro

$\begin{array}{llll}\left.\right|_{\text {Trp9Arg }} ^{A} & \text { Asn247Ser } & \text { Tyr425Asn } & \text { Leu574Pro }\end{array}$

Four of the base pair changes are silent; the alteration in the amino acids are indicated (underlined). For clarity in the text, the altered amino acids in the mutant, Trp9Arg, Asn247Ser, Tyr425Asn and Leu574Pro have been termed mutations A, B, $\mathrm{C}$ and $\mathrm{D}$, respectively and are shown in the schematic diagram

Site-directed mutagenesis. To simplify the mutation nomenclature, the four amino-acid mutations in the mutant $\mathrm{GAD}_{65}$, Trp9Arg, Asn247Ser, Tyr425Asn and Leu574Pro were termed mutations A, B, C and D, respectively (Table 1 ). Thus, we have denoted the mutant $\mathrm{GAD}_{65}$ with all four mutations mutant $\mathrm{GAD}_{65} / \mathrm{ABCD}$. The mutant $\mathrm{GAD}_{65}$ was subcloned into pALTER-1 (Promega, Southampton, UK) and site-directed

Fig.1. Sequences and annealing sites of the mutagenic oligonucleotides used to revert the altered amino acids in mutant $\mathrm{GAD}_{65}$ to those found in the wild-type protein. The sequence is numbered from the first nucleotide of the innitiation methionine. Altered amino acids are shown in bold type. New restriction sites created are underscored and the corresponding enzymes AvaII, EcoRV, NheI and EcoNI shown below mutagenesis was done using the Altered Sites II kit (Promega) according to the manufacturer's instructions. The mutagenic oligonucleotides are shown in Fig. 1 and the presence of the desired mutations confirmed by DNA sequencing. To analyse the role of each of the four alterations in mutant $\mathrm{GAD}_{65} / \mathrm{ABCD}$, a number of mutagenesis reactions were done in which one, two or three of the alterations in the mutant were reverted back to the wild-type amino acids. For example, $\mathrm{GAD}_{65}$ containing only the single amino-acid mutation $\mathrm{D}$ was generated by reverting amino acids $\mathrm{A}, \mathrm{B}$ and $\mathrm{C}$ in the mutant $\mathrm{GAD}_{65} / \mathrm{ABCD}$ to wild-type residues. This mutant was termed mutant $\mathrm{GAD}_{65} / \mathrm{D}$. Similarly, the double mutant containing only mutation $\mathrm{B}$ and mutation $\mathrm{D}$ was generated by reverting residues $\mathrm{A}$ and $\mathrm{C}$ to wild-type residues and termed mutant $\mathrm{GAD}_{65} / \mathrm{BD}$.

Antibody analysis. Wild-type $\mathrm{GAD}_{65}$ and all mutant $\mathrm{GAD}_{65}$ cDNAs were subcloned into pGEM4-Z (Promega) and protein produced by transcription translation in vitro in a singletube reaction using a TNT T7 quick-rabbit reticulocyte lysate system in the presence of ${ }^{35} \mathrm{~S}$-methionine. The size of all translated proteins was confirmed by SDS polyacrylamide gel electrophoresis and autoradiography. The expression of translated products of wild-type $\mathrm{GAD}_{65}, \mathrm{GAD}_{67}$ and all mutant $\mathrm{GAD}_{65}$ was monitored using serum from an SMS patient containing high-titre anti-GAD 65 antibodies. The conformational stability of the translated products was confirmed by reactivity with a panel of $\mathrm{GAD}_{65}$ antibody-positive sera from Type I diabetic patients. All translated products gave similar reactivity to control antibodies when either used freshly prepared (without freezing) in shorter incubations or after freezing and in overnight incubations with antibodies. Immunoprecipitations were done at $4-7^{\circ} \mathrm{C}$ in 96 -well microtitre plates using MultiScreen filtration plates (Millipore, Watford, UK) as described [36]. Data presented for human sera and hmABs are the mean of duplicate samples. Background binding [binding to 20 normal healthy control sera, GAD antibody negative culture supernatants or protein A Sepharose alone (Amersham-Pharmacia Biotech, Little Chalfont, UK)] was subtracted from the binding of the serum or hmAB-containing culture supernatant.
9

5

Mutant GAD65

Primer A

GlySerGlyPheArgSerPheGlySerGlu GGGCTCTGGCTTTCGGTCTTTCGGGTCGGAAG ||||||||||||x||||x|||||||||||| CCCGAGACCGAAAACCAGGAAGCCCAGCCTTC

Trp

AvaII

247

240 SerProGlyGlyAlaIleSerserMetTyrAlaMet

Mutant GAD65

Primer B

Mutant GAD65

Primer C

Mutant GAD65

Primer D
TCTCCCGGTGGCGCCATATCTAGCATGTATGCCATGAT
||||||||||||||||||||$|x|||||||||||||$
AGAGGGCCACCGCGCTATAGATTGTACATACGGTACTA

AGAGGGCCACCGCGCTATAGATTG
Asn

ECORV

425

418 CysAsnglnMetHisAlaSerAsnLeuPheGlnGln TGCAACCAAATGCATGCCTCCAACCTCTTTCAGCAA $|\|\|\|\|\|\|\||| x|| x x|x| \mid\|\|\|\|\|\|\| \|$ ACGTTGGTTTACGTGCGATCGATGGAGAAAGTCGTT

$$
\text { NheI }
$$

574

570

AspIleAspPheProIl

GACATTGACTTCCCGATTGAAGAAATAGAACGCC

||||||||||||x||||||x|||||||||||

CTGTAACTGAAGGACTAACTCCTTTATCTTGCGG

Leu
14

252

429

ECONI 
The binding of the hmABs to $\mathrm{GAD}_{65}$ mutants was considered to be substantially affected if the reactivity represented $5-25 \%$ of that precipitated with wild-type $\mathrm{GAD}_{65}$; the antibodies were considered to bind weakly if the reactivity represented $25-40 \%$ of wild-type binding. The results of the binding of serum antibodies to mutant $\mathrm{GAD}_{65}$ were analysed by MannWhitney $\mathrm{U}$ test. We considered $p$ values of less than 0.05 significant.

Expression of recombinant $G A D_{65}$ in insect cells. Recombinant baculovirus was produced using BaculoGold transfection kit with mutant GAD $_{65}$ cDNAs subcloned into pVL1393 (Becton-Dickinson, Oxford, UK). As control, wild-type $\mathrm{GAD}_{65}$ recombinant baculovirus (from Dr T. Dyrberg) [37] was expressed in parallel Sf9 insect cells. Expression of recombinant $\mathrm{GAD}_{65}$ proteins in Sf9 cells was monitored by SDS polyacrylamide gel electrophoresis and western blotting with rabbit antisera generated to $\mathrm{GAD}_{65}$ peptides (serum 6952 and 7996 to GAD $_{65}$ peptides 4-22 and 570-585, respectively from $\mathrm{Dr} \AA$. Lernmark) [38]. The enzymatic activity of insect-cell mutant $\mathrm{GAD}_{65}$ was measured in homogenates of GAD expressing insect cells by the release of ${ }^{14} \mathrm{CO}_{2}$ from its substrate, ${ }^{14} \mathrm{C}$-glutamic acid as described [39] and compared with the activity of wildtype $\mathrm{GAD}_{65}$.

\section{Results}

Cloning of mutant $G A D_{65}$. We cloned $\mathrm{GAD}_{65}$ from human islet total cDNA by double PCR amplification from total human islet cDNA. Sequencing of both strands showed eight base-pair alterations from the published sequence, with four of these alterations leading to a change in the amino acids Trp9Arg (mutation A), Asn247Ser (mutation B), Tyr425Asn (mutation C) and Leu574Pro (mutation D) [2, 3] (Table 1). This mutant was termed mutant $\mathrm{GAD}_{65} /$ $A B C D$. We investigated whether the mutant $\mathrm{GAD}_{65} / \mathrm{ABCD}$ arose as a result of incorrect basepair incorporation by the Taq polymerase [40] during the PCR reaction. The sequences around three of the base-pair alterations, nucleotides 740 (mutation B), 1273 (mutation C) and 1721 (mutation D) were amplified using the original stock of human islet cDNA used in the cloning of mutant $\mathrm{GAD}_{65}$. Sequencing of more than ten independent clones of each of the mutants showed a complete concordance of the sequence with wild-type $\mathrm{GAD}_{65}$ giving confirmatory evidence that the alterations in mutant $\mathrm{GAD}_{65}$ derive from incorrect base-pair incorporation during the PCR reaction. Notably three of the amino-acid substitutions, at positions 247 (mutation B), 425 (mutation C) and 574 (mutation D) are also conserved in the related human isoform, $\mathrm{GAD}_{67}[2,3]$; furthermore, all four altered residues in the mutant are conserved in the murine and porcine $\mathrm{GAD}_{65}[41,42]$, suggesting important roles in protein folding or function.

Binding of serum antibodies to mutant $G A D_{65}$. When binding of $\mathrm{GAD}_{65}$ antibody-positive sera from new-

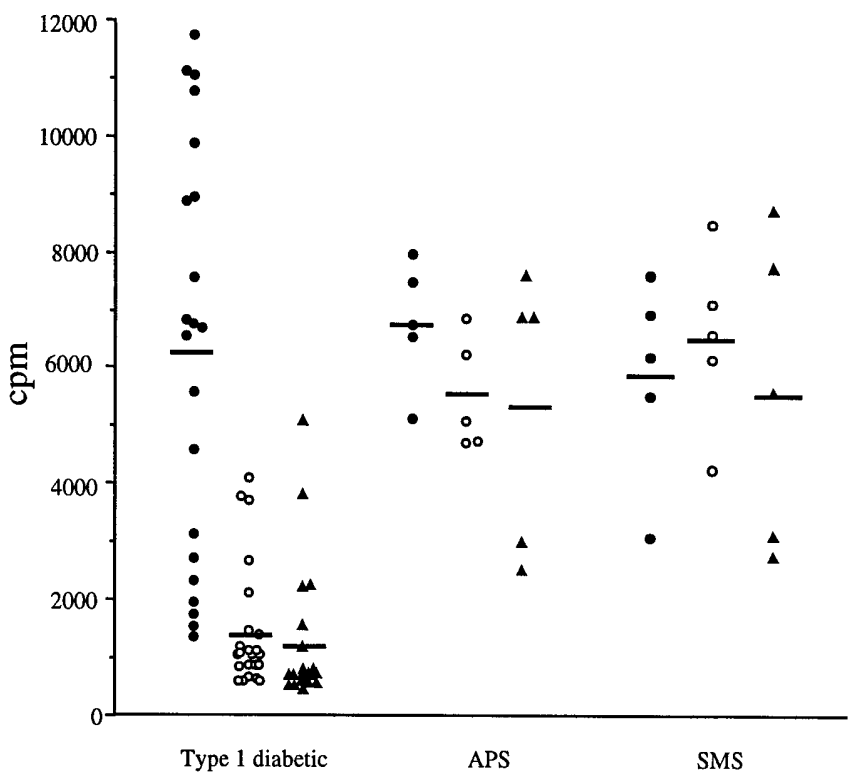

Fig. 2. Immunoprecipitation analyses of wild-type $\mathrm{GAD}_{65}$ (O), mutant $\mathrm{GAD}_{65} / \mathrm{ABCD}(\mathrm{O})$ and $\operatorname{GAD}_{67}(\boldsymbol{\Delta})$ with $\mathrm{GAD}_{65}$ antibody sera from new-onset Type I diabetic, APS and SMS patients using ${ }^{35} \mathrm{~S}$-methionine-labelled, translated proteins. Binding of individual sera from different patient groups to various GAD proteins was analysed using the Mann-Whitney U test. The bars represent the group mean values for patients sera precipitated with the translated proteins. The binding of Type I diabetic sera to mutant $\mathrm{GAD}_{65} / \mathrm{ABCD}$ is significantly reduced compared with the reactivity with wild-type $\mathrm{GAD}_{65}$. There was no significant difference in the binding of $\mathrm{GAD}_{65}$-positive APS and SMS patients sera with the mutant and wild-type $\mathrm{GAD}_{65}$

onset Type I diabetic patients to mutant $\mathrm{GAD}_{65} /$ ABCD and wild-type $\mathrm{GAD}_{65}$ was compared, a striking difference was apparent in which substantially lower binding to the mutant was observed for all sera (group mean mutant $\mathrm{GAD}_{65}=1380 \mathrm{cpm}$, wildtype $\left.\mathrm{GAD}_{65}=6147 \mathrm{cpm}\right)(p<0.0001)$ (Fig. 2). No such difference between the binding to mutant $\mathrm{GAD}_{65} / \mathrm{ABCD}$ and wild-type $\mathrm{GAD}_{65}$ was observed, however, in sera from APS patients (group mean mutant $\mathrm{GAD}_{65}=5514 \mathrm{cpm}$, wild-type $\mathrm{GAD}_{65}=6760$ cpm) $(p>0.05)$ or SMS patients (group mean mutant $\mathrm{GAD}_{65}=6538 \mathrm{cpm}$, wild-type $\mathrm{GAD}_{65}=5904 \mathrm{cpm}$ ) $(p>0.05)$. The data indicate that one or more of the amino-acid alterations in mutant $\mathrm{GAD}_{65} / \mathrm{ABCD}$ results in a statistically significant alteration in the binding of the majority of serum antibodies from Type I diabetic patients. The titres of anti-GAD ${ }_{65}$ antibodies in APS and SMS patients are considerably higher than those in sera from Type I diabetic patients [17-20]. To exclude the possibility that higher titres of anti-GAD ${ }_{65}$ antibodies in APS and SMS sera were responsible for the binding to mutant $\mathrm{GAD}_{65} /$ ABCD, we diluted APS and SMS sera so that they precipitated similar counts of translated wild-type $\mathrm{GAD}_{65}$ to those precipitated by Type I diabetic sera. 


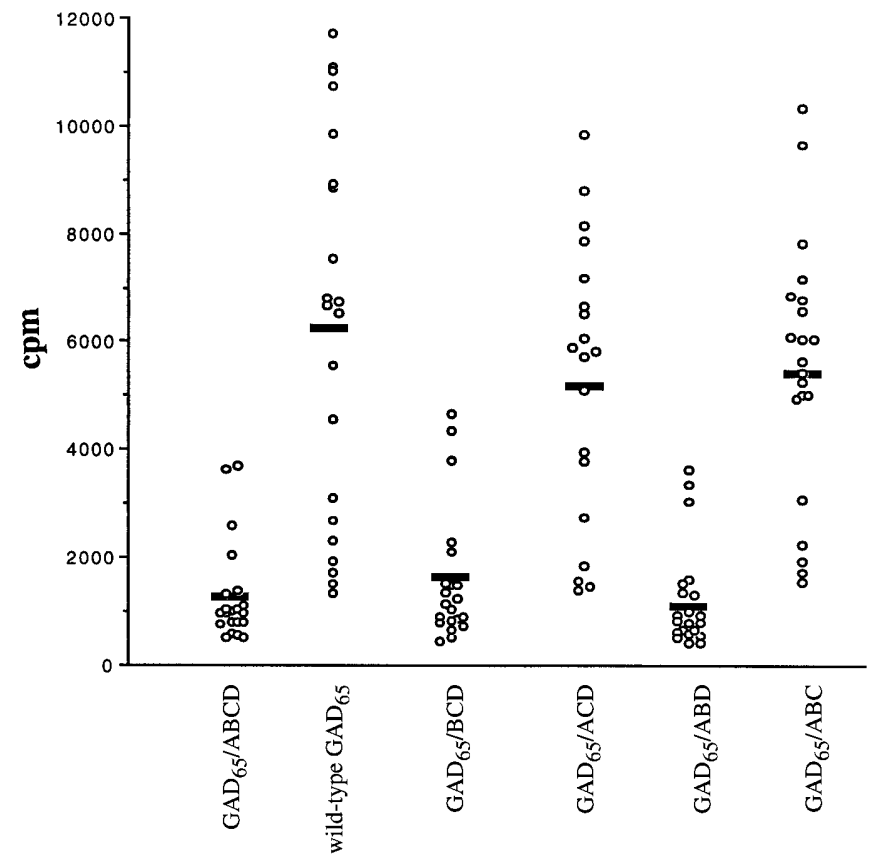

Fig. 3. Immunoprecipitation analyses of $\mathrm{GAD}_{65}$ antibody sera from new-onset Type I diabetic patients with different mutants of $\mathrm{GAD}_{65}$ using ${ }^{35} \mathrm{~S}$-methionine-labelled, translated proteins. The reactivity of $\mathrm{GAD}_{65}$ antibodies in the Type I diabetic sera is restored in the single amino-acid mutants $\mathrm{GAD}_{65} / \mathrm{ACD}$ and $\mathrm{GAD}_{65} / \mathrm{ABC}$ indicating that substitution $\mathrm{B}$ and substitution $\mathrm{D}$ are responsible for the loss of binding with the mutant $\mathrm{GAD}_{65} / \mathrm{ABCD}$. The bars represent the group mean values

These diluted sera still reacted with the mutant $\mathrm{GAD}_{65} / \mathrm{ABCD}$ showing that this effect was not related to antibody titres (not shown). The binding of sera to $\mathrm{GAD}_{67}$ was also assessed. Sera from Type I diabetic patients showed weak binding to $\mathrm{GAD}_{67}$ and no statistically significant correlation was observed to levels of binding to either wild-type $\mathrm{GAD}_{65}$ or mutant $\mathrm{GAD}_{65} / \mathrm{ABCD}$. In contrast, sera from APS and SMS patients showed levels of binding to $\mathrm{GAD}_{67}$ similar to wild-type $\mathrm{GAD}_{65}$ (Fig. 2).

Effect of reversion of single amino-acid replacements of mutant $G A D_{65} / A B C D$ to wild-type residue. To assess the amino acid(s) in mutant $\mathrm{GAD}_{65} / \mathrm{ABCD}$ responsible for the loss of binding of $\mathrm{GAD}_{65}$ antibodies in Type I diabetic sera, each of the four mutant amino acids was individually reverted back to wild-type residue, creating four new mutants termed mutant $\mathrm{GAD}_{65} / \mathrm{BCD}, \quad \mathrm{GAD}_{65} / \mathrm{ACD}, \mathrm{GAD}_{65} / \mathrm{ABD}$ and $\mathrm{GAD}_{65} / \mathrm{ABC}$. Translation products from all mutants were found to co-migrate with wild-type $\mathrm{GAD}_{65}$ in SDS polyacrylamide gel electrophoresis (not shown). Binding of sera from Type I diabetic patients showed higher levels of binding to mutant $\mathrm{GAD}_{65} / \mathrm{ACD}$ $($ mean $=5000 \mathrm{cpm}, p<0.0001)$ and mutant $\mathrm{GAD}_{65} /$ $\mathrm{ABC}($ mean $=5483, p<0.0001)$ compared with mutant $\mathrm{GAD}_{65} / \mathrm{ABCD}$ (Fig. 3). Binding to mutant
$\mathrm{GAD}_{65} / \mathrm{BCD} \quad($ mean $=1571, p>0.05)$ and mutant $\mathrm{GAD}_{65} / \mathrm{ABD}$ (mean $\left.=1210, p>0.05\right)$ was, however, similar to mutant $\mathrm{GAD}_{65} / \mathrm{ABCD}$ (Fig. 3). The higher levels of antibody binding from Type I diabetic sera observed when either residues B or D were reverted to wild-type residues suggests that mutations B and $\mathrm{D}$ were responsible for the loss of antibody reactivity to mutant $\mathrm{GAD}_{65} / \mathrm{ABCD}$; in contrast, mutations $\mathrm{A}$ or $\mathrm{C}$ have minimal influence on antibody binding in the mutant $\mathrm{GAD}_{65} / \mathrm{ABCD}$.

To focus on the critical roles of mutations B and D in antibody binding from Type I diabetic sera, new mutants were generated which contained only these mutations either singly or in combination, i. e. mutant $\mathrm{GAD}_{65} / \mathrm{B}, \mathrm{GAD}_{65} / \mathrm{D}, \mathrm{GAD}_{65} / \mathrm{BD}$ and were used in immunoprecipitation experiments with Type I diabetic sera. The binding of sera to mutant $\mathrm{GAD}_{65} / \mathrm{BD}$ was similar to that observed to mutant $\mathrm{GAD}_{65} /$ $\mathrm{ABCD}$ whereas binding to mutant $\mathrm{GAD}_{65} / \mathrm{B}$ and $\mathrm{GAD}_{65} / \mathrm{D}$ was statistically significantly higher, confirming the importance of mutations in residues $\mathrm{B}$ or $\mathrm{D}$ for loss of antibody binding (data not shown).

Binding of hmABs to single and double amino-acid mutants of $G A D_{65}$. As the $\mathrm{GAD}_{65}$ autoantibodies in Type I diabetic sera are heterogeneous and recognise a variety of conformational-dependent determinants on $\mathrm{GAD}_{65}$, we used hmABs to $\mathrm{GAD}_{65}$ to determine the role of mutations $\mathrm{B}$ and $\mathrm{D}$ in maintaining the integrity of conformational-dependent regions in $\mathrm{GAD}_{65}$. A panel of $11 \mathrm{hmABs}$, where all (except MICA 2) are dependent on recognition of different discontinous determinants on $\mathrm{GAD}_{65}$, were used $[27-33,43]$. None of the hmABs react with $\mathrm{GAD}_{67}$ $[28,29,31]$. The binding of the hmABs with the mutant $\mathrm{GAD}_{65} / \mathrm{ABCD}$ was either abolished (DP A, DP B, DP D and MICA 3) ( < 10\% binding of wild-type $\mathrm{GAD}_{65}$ binding) or statistically significantly reduced (MICA 1, MICA 2, MICA 4, MICA 6, DP C, b78 and b96) (10-25\% binding of wild-type $\mathrm{GAD}_{65}$ ). There was similar reduced binding to the double amino-acid mutant, $\mathrm{GAD}_{65} / \mathrm{BD}$ with all the hmABs (Fig. 4). Analysis of the binding to the single aminoacid mutants, $\mathrm{GAD}_{65} / \mathrm{B}$ and $\mathrm{GAD}_{65} / \mathrm{D}$ showed two patterns of binding. All the hmABs show reactivity with the mutant $\mathrm{GAD}_{65} / \mathrm{B}$ and thus are not influenced by the single amino-acid mutation $B$ in $\mathrm{GAD}_{65}$. In contrast, the binding to the other single amino-acid mutant, GAD 65 D with MICA 1 and DP A was statistically significantly effected; MICA 3 showed reduced reactivity with this mutant. Notably, all these three $\mathrm{hmABs}$ are dependent on C-terminal epitopes of $\mathrm{GAD}_{65}$ [33]. The binding of the remaining hmABs with the mutant $\mathrm{GAD}_{65} / \mathrm{D}$, including other C-terminal-directed hmABs such as MICA 2 and b78, was not significantly effected ( $>60 \%$ binding of wild-type $\mathrm{GAD}_{65}$ ) (Fig. 4). In summary, all eleven hmABs to $\mathrm{GAD}_{65}$ show a loss of binding to mutant 

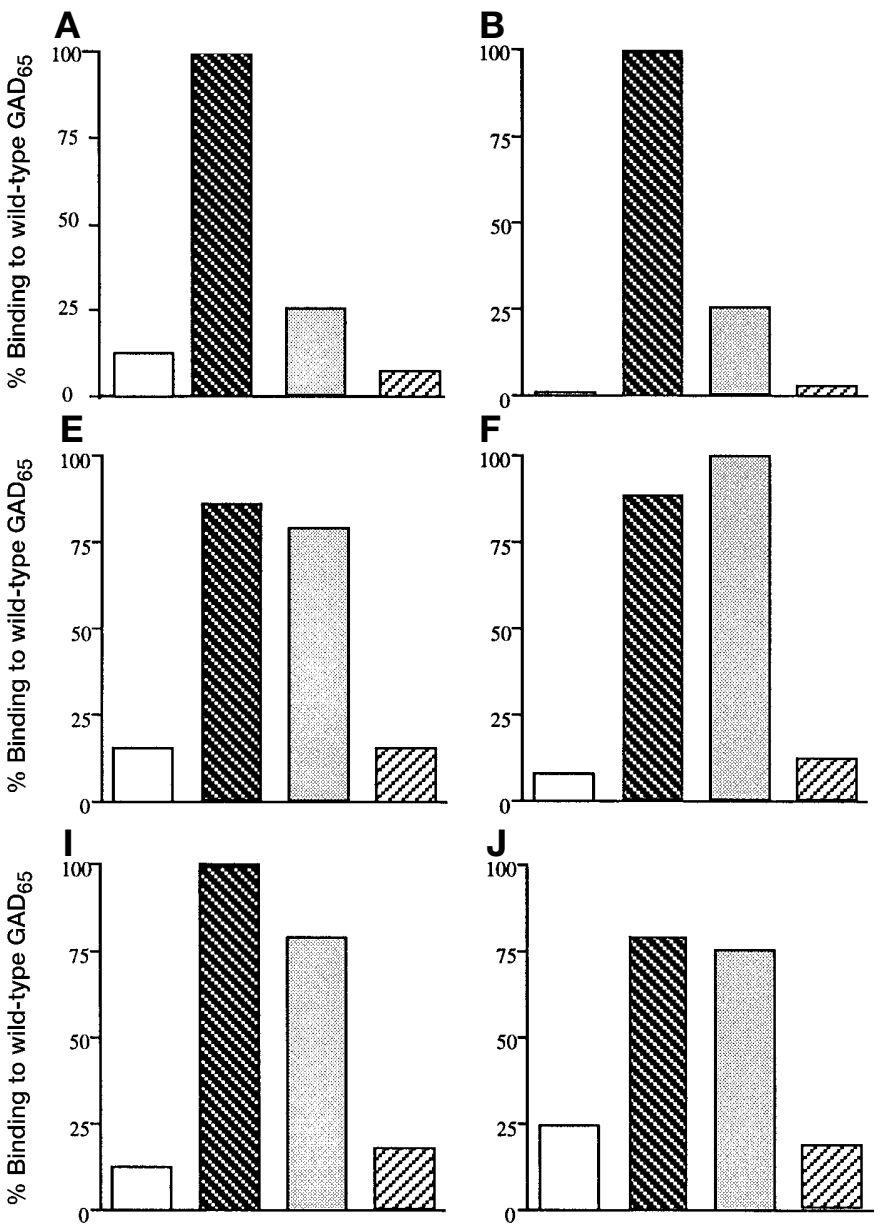
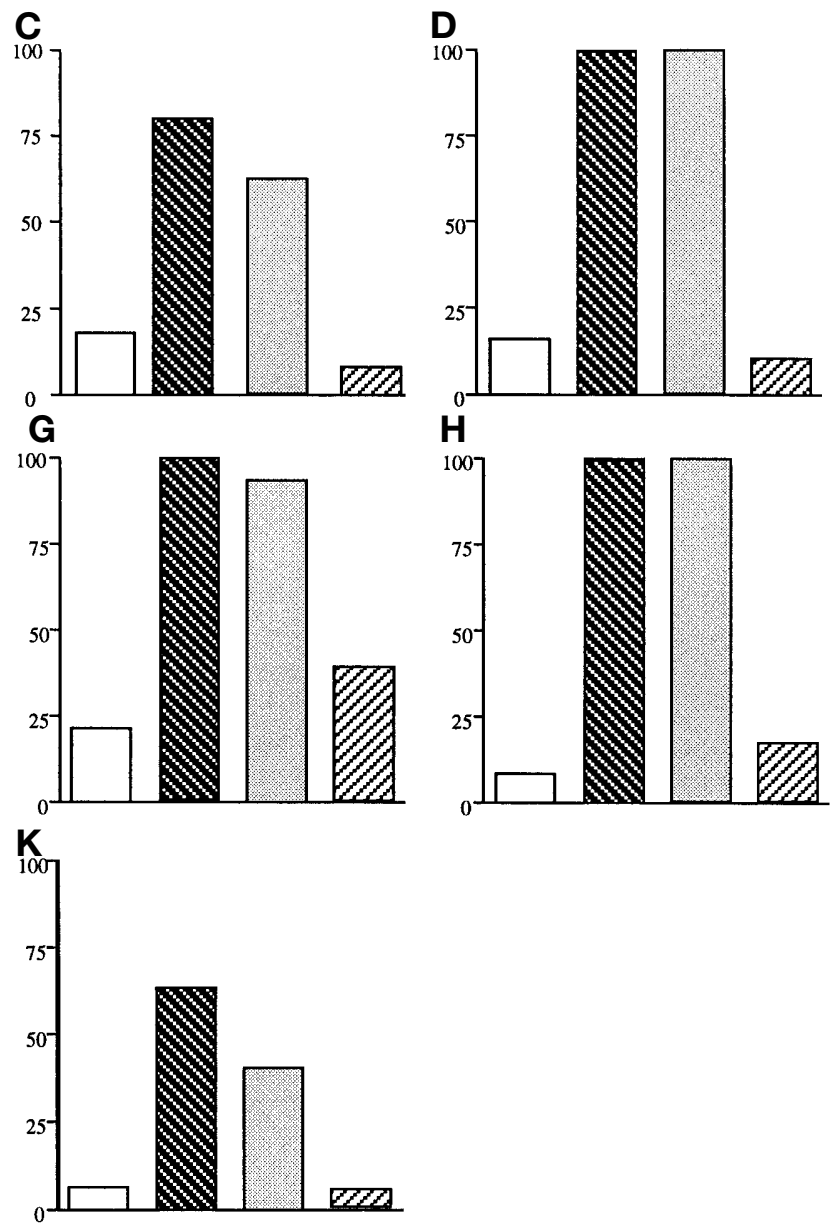

Fig.4A-K. Immunoprecipitation analyses of eleven hmABs (A MICA 1, B DP A, C MICA 2, D MICA 4, E MICA 6, F DP B, G DP C, H DP D, I b78, J b96, K MICA 3) with mutants of $\mathrm{GAD}_{65}$ using ${ }^{35} \mathrm{~S}$-methionine-labelled, translated proteins. The results are expressed as percentage values of wild-type $\mathrm{GAD}_{65}$ precipitated in the same experiment. The GAD mutants used are as follows: $\mathrm{GAD}_{65} / \mathrm{ABCD}(\square) ; \mathrm{GAD}_{65} / \mathrm{B}(\mathbb{N})$; $\mathrm{GAD}_{65} / \mathrm{D}(\square)$ and $\mathrm{GAD}_{65} / \mathrm{BD}(\mathbb{V})$. The experiments have been repeated at least three times with similar results. In particular, the hmABs MICA 1, MICA 3 and DP-A which are influenced by mutation $\mathrm{D}$, the reduction in percentage binding to mutant $\mathrm{D}$ compared with wild-type $\mathrm{GAD}_{65}$ binding was between $6-24 \%, 38-39 \%$ and $11-26 \%$, respectively. None of the $\mathrm{hmABs}$ react with $\mathrm{GAD}_{67}$ run in a parallel experiment (not shown)

$\mathrm{GAD}_{65} / \mathrm{BD}$. Mutation $\mathrm{B}$ on its own in mutant $\mathrm{GAD}_{65} / \mathrm{B}$ did not statistically significantly effect the binding of any of the hmABs but mutation $\mathrm{D}$ in mutant $\mathrm{GAD}_{65} / \mathrm{D}$ resulted in the loss of binding of a small number of hmABs. Therefore, the simultaneous presence of mutations $\mathrm{B}$ and $\mathrm{D}$ together in mutant $\mathrm{GAD}_{65} / \mathrm{BD}$ was necessary to disrupt the binding of conformationally dependent hmABs to $\mathrm{GAD}_{65}$.

Expression of recombinant mutant $G A D_{65}$. The mutants, $\mathrm{GAD}_{65} / \mathrm{ABCD}, \mathrm{GAD}_{65} / \mathrm{BD}$ and $\mathrm{GAD}_{65} / \mathrm{D}$ were expressed as recombinant proteins with baculovirus in insect cells. All mutant $\mathrm{GAD}_{65}$ recombinant proteins co-migrated in SDS polyacrylamide gels with the control, wild-type $\mathrm{GAD}_{65}$ expressed in insect cells (not shown). None of the mutants showed decarboxylase enzymatic activity; replacement of residues $\mathrm{A}, \mathrm{B}, \mathrm{C}$ and $\mathrm{D}$ in the mutant with those present in wild-type $\mathrm{GAD}_{65}$ led to restoration of decarboxylase activity, which matched the control $\mathrm{GAD}_{65}$ expressed in insect cells [37] (not shown).

\section{Discussion}

Our study reports on a fortuitous finding on a mutant $\mathrm{GAD}_{65}$ that arose as a result of random mutations during PCR cloning of the molecule. The mutant $\mathrm{GAD}_{65}$ arose as a result of fortuitous incorrect base incorporations due to the use of Taq polymerase [40]. There are differences between the antibody specificities to $\mathrm{GAD}_{65}$ present in new-onset Type I diabetic patients and those in APS and SMS patients, with the former antibodies recognising predominantly conformational-dependent epitopes on $\mathrm{GAD}_{65}$. [6, 13, 18-26]. The preferential loss of binding of anti- 
bodies from new-onset Type I diabetic sera to the mutant $\mathrm{GAD}_{65}$ suggests that the mutations probably effect the surface conformation and hence the three-dimensional folding of the molecule. The replacement of two residues present in the mutant $\mathrm{GAD}_{65}$, Asn247 (residues B) and Leu574 (residue D) was necessary to restore the binding of the autoantibodies of Type I diabetic sera, indicating the crucial nature of these two amino acids in maintaining the structural integrity of $\mathrm{GAD}_{65}$.

It was remarkable that two single amino-acid replacements in $\mathrm{GAD}_{65}$ were able to discriminate the multiple antibody specificities to $\mathrm{GAD}_{65}$ present in sera from Type I diabetic patients from those of APS and SMS sera. The preferential binding of $\mathrm{GAD}_{65}$ antibodies in APS and SMS sera with the mutant $\mathrm{GAD}_{65}$ was not related to the lower antibody titres generally found in Type I diabetic sera [17-20]. To simplify the complex patterns of serum anti-GAD 65 antibody specificities, the role of the two replacements was clarified using hmABs to $\mathrm{GAD}_{65}$. The finding that all eleven $\mathrm{hmABs}$ used in this study, which recognise different determinants localised to the N-region, C-region and the middle region of $\mathrm{GAD}_{65}$ [28-33], loose their binding to the double mutant Asn247Ser and Leu574Pro suggests that the two repacements have a considerable effect on the overall surface conformation of the entire molecule.

The single amino-acid replacements at residues 247 (position B) or 574 (position D) of $\mathrm{GAD}_{65}$ was found to influence the binding of the hmABs differentially. Whereas the replacement of residue 247 was found to be silent in terms of reactivity of the $\mathrm{hmABs}$, the replacement of residue 574 influenced the binding of some hmABs from the panel, including MICA 1, MICA 3 and DP-A. All these three hmABs are directed to the C-terminal region of $\mathrm{GAD}_{65}$ [33], but notably other C-terminal-directed hmABs such as MICA 2 and b78 [30-33], were not affected in their reactivity to the single amino-acid replacement in the mutant $\mathrm{GAD}_{65}$. Recent mapping of the determinants recognised by MICA 1, MICA 3 and DP-A on GAD $_{65}$ indicate that they recognise three distinct, but similar, determinants which are dependent on residues 483-499 and residues 556-585 [33], whereas the determinants recognised by MICA 2 and b78 reside in distinct residues 512-540 and $514-570$, respectively $[32,43]$. It is likely that residue 574 forms a contact amino acid for MICA 1, MICA 3 and/or DP-A or it has an effect on the conformation that is localised to the extreme $\mathrm{C}$-terminal region of the molecule without affecting the nearby regions of amino acids 512-540 recognised by MICA 2 and b78. Other single amino acid substitutions in $\mathrm{GAD}_{65}$ have been described but their effects appear to be limited to influencing the binding of some hmABs [33], as described above for residue 574. A three-dimensional model of the middle and the C-terminal domain of the $\mathrm{GAD}_{65}$ dimer has been constructed based on structure modelling from other decarboxylases whose structure is known [33]. The model indicates that the middle and the $\mathrm{C}$-terminal regions form two very separate folding domains in the $\mathrm{GAD}_{65}$ model. Notably, the two mutations identified in this study that lead to destabilisation of the $\mathrm{GAD}_{65}$ structure reside in predicted helices (helix I and helix $\mathrm{T}$ ) which are buried in the two domains [33], raising the possibility that the simultaneous disruption of both the folded domains leads to total destabilisation of the $\mathrm{GAD}_{65}$ structure whereas single replacement of these residues either has minimal (residue 247) or limited (residue 574) local conformational effects on the molecule.

The concerted effect of two amino acids Asn-247 and Leu-574 in GAD $_{65}$ is essential for maintenance of the conformational determinants recognised by anti-GAD65 autoantibodies directed to a variety of discontinous epitopes, as well as the maintainance of functional enzymatic activity of the molecule. The replacements of these two residues could be useful in discriminating autoimmune sera from Type I diabetic patients from those of APS and SMS by immunoprecipitation assay. In conjunction with $\mathrm{GAD}_{67}$ precipitation, ambiguous sera could also be confirmed with the use of the two mutants. It is also possible that some changes in epitope patterns of anti-GAD ${ }_{65}$ antibodies during progression to disease activity in Type I diabetes could be monitored with the two mutants [44].

Acknowledgements. We thank Dr M. Christie for advice on the measurement of decarboxylase enzymatic activity. We are grateful to Drs T. Dyrberg and Å. Lernmark for donating recombinant $\mathrm{GAD}_{65}$ baculovirus and anti-GAD peptide antisera, respectively. This project was funded by the British Diabetes Association.

\section{References}

1. Erlander MG, Tobin AJ (1991) The structural and functional heterogeneity of glutamic acid decarboxylase: a review. Neurochem Res 16: 215-226

2. Karlsen AE, Hagopian WA, Grubin CE et al. (1991) Cloning and primary sequence of a human islet isoform of glutamic acid decarboxylase from chromosome 10. Proc Natl Acad Sci USA 88: 8337-8341

3. Bu D, Erlander MG, Hitz BC et al. (1992) Two human glutamate decarboxylases, $65 \mathrm{kDa}$ GAD and $67 \mathrm{kDa}$ GAD, are each encoded by a single gene. Proc Natl Acad Sci USA 89: 2115-2119

4. Bu D, AJ Tobin (1994) The exon-intron organisation of the genes (GAD1 and GAD2) encoding two human glutamate decarboxylases (GAD67 and GAD65) suggests that they derive from a common ancestral GAD. Genomics 21: 222-228

5. Kim J, Richter W, Aanstoot HJ et al. (1993) Differential expression of GAD65 and GAD67 in human, rat, and mouse pancreatic islets. Diabetes 42: 1799-1803 
6. Baekkeskov S, Aanstoot HJ, Christgau S et al. (1990) Identification of the $64 \mathrm{~K}$ autoantigen in insulin-dependent diabetes as the GABA-synthesizing enzyme glutamic acid decarboxylase. Nature 347: 151-156

7. Christie MR (1996) Antibodies to glutamic acid decarboxylase in the prediction of insulin dependent diabetes. In: Palmer J (ed) Diabetes; Prediction, Prevention and Genetic Councelling. John Wiley and Sons, Chichester, pp 77-96

8. Lernmark $\AA$ (1996) Glutamic acid decarboxylase - gene to antigen to disease. J Intern Med 240: 259-277

9. Solimena M, De Camilli P (1991) Autoimmunity to glutamic acid decarboxylase (GAD) in Stiff Man Syndrome and insulin dependent diabetes mellitus. Trends Neurosci 14: 452-457

10. Atkinson MA, Kaufman DL, Newman D, Tobin AJ, Maclaren NK (1993) Islet cell cytoplasmic autoantibody reactivity to glutamate decarboxylase in insulin-dependent diabetes. J Clin Invest 91: 350-356

11. Kaufman DL, Erlander MG, Clare-Salzler M, Atkinson MA, Maclaren NK, Tobin AJ (1992) Autoimmunity to two forms of glutamate decarboxylase in insulin-dependent diabetes mellitus. J Clin Invest 89: 283-292

12. Seissler J, Amann J, Mauch L et al. (1993) Prevalence of autoantibodies to the 65 - and $67-\mathrm{kD}$ isoforms of glutamate decarboxylase in insulin-dependent diabetes mellitus. J Clin Invest 92: 1394-1399

13. Tuomi T, Bjorses P, Falorni A et al. (1996) Antibodies to glutamic acid decarboxylase and insulin-dependent diabetes in patients with autoimmune polyendocrine syndrome type I. J Clin Endocrinol Metab 81: 1488-1494

14. Davenport C, Radford PM, Al-Burhari T, Lai M, Bottazzo GF, Todd I (1998) Heterogeneity in the occurrence of a subset of autoantibodies to glutamic acid decarboxylase in autoimmune polyendocrine patients with islet cell antibodies. Clin Exp Immunol 111: 497-505

15. Hagopian WA, Michelsen B, Karlsen AE et al. (1993) Autoantibodies in IDDM primarily recognize the 65,000$\mathrm{M}(\mathrm{r})$ rather than the $67,000-\mathrm{M}(\mathrm{r})$ isoform of glutamic acid decarboxylase. Diabetes 42: 631-636

16. Falorni A, Ackefors M, Carlberg C et al. (1996) Diagnostic sensitivity of immunodominant epitopes of glutamic acid decarboxylase (GAD65) autoantibodies in childhood IDDM. Diabetologia 39: 1091-1098

17. Christie MR, Genovese S, Cassidy D et al. (1994) Antibodies to islet $37 \mathrm{k}$-antigen, but not to glutamate decarboxylase, discriminate rapid progression to insulin-dependent diabetes mellitus in endocrine autoimmunity. Diabetes 43: 1254-1259

18. Solimena M, Folli F, Aparisi R, Pozza G, De Camilli P (1990) Autoantibodies to GABA-ergic neurons and pancreatic beta cells in stiff-man syndrome. New Engl J Med 322: $1555-1560$

19. Seissler J, Beig S, Yassin N et al. (1994) Association between antibodies to the MR 67, 000 isoform of glutamate decarboxylase (GAD) and type 1 (insulin-dependent) diabetes mellitus with coexisting autoimmune polyendocrine syndrome type II. Autoimmunity 19: 231-238

20. Kim J, Namchuk M, Bugawan T et al. (1994) Higher autoantibody levels and recognition of a linear $\mathrm{NH}_{2}$-terminal epitope in the autoantigen GAD65 distinguish GAD65 distinguish stiff-man syndrome from insulin-dependent diabetes mellitus. J Exp Med 180: 595-606

21. Ujihara N, Daw K, Gianani R, Boel E, Yu L, Powers AC (1994) Identification of glutamic acid decarboxylase autoantibody heterogeneity and epitope regions in type I diabetes. Diabetes 43: 968-975
22. Daw K, Ujihara N, Atkinson M, Powers AC (1996) Glutamic acid decarboxylase autoantibodies in stiff-man syndrome and insulin-dependent diabetes mellitus exhibit similarities and differences in epitope recognition. J Immunol 156: 818-825

23. Butler MH, Solimena M, Dirkx R, Hayday A, De Camilli P (1993) Identification of a dominant epitope of glutamic acid decarboxylase (GAD-65) recognized by autoantibodies in stiff-man syndrome. J Exp Med 178: 2097-2106

24. Li L, Hagopian WA, Brashear R, Daniels T, Lernmark Å (1993) Identification of a dominant epitope of glutamic acid decarboxylase (GAD-65) recognized by autoantibodies in stiff-man syndrome. J Immunol 152: 930-934

25. Bjork E, Velloso LA, Kampe O, Karlsson A (1994) GAD autoantibodies in IDDM, Stiff-man syndrome, and Autoimmune Polyendocrine Syndrome Type I recognize different epitopes. Diabetes 43: 161-165

26. Dinkel K, Meinck HM, Jury KM, Karges W, Richter W (1998) Inhibition of gamma-aminobutyric acid synthesis by glutamic acid decarboxylase autoantibodies in stiff-man syndrome. Ann Neurol 44: 194-201

27. Richter W, Endl J, Eiermann TH et al. (1992) Human monoclonal islet cell antibodies from a patient with insulin-dependent diabetes mellitus reveal glutamate decarboxylase as the target antigen. Proc Natl Acad Sci USA 89: $8467-8471$

28. Richter W, Shi Y, Baekkeskov S (1993) Autoreactive epitopes defined by diabetes-associated human monoclonal antibodies are localized in the middle and C-terminal domains of the smaller form of glutamate decarboxylase. Proc Natl Acad Sci USA 90: 2832-2836

29. Madec AM, Rousset F, Ho S et al. (1996) Four IgG anti-islet human monoclonal antibodies isolated from a type $1 \mathrm{di}-$ abetes patient recognize distinct epitopes of glutamic acid decarboxylase 65 and are somatically mutated. J Immunol 156: 3541-3549

30. Syren K, Lindsay L, Stoehrer B et al. (1996) Immune reactivity of diabetes-associated human monoclonal autoantibodies defines multiple epitopes and detects two domain boundaries in glutamate decarboxylase. J Immunol 157: 5208-5214

31. Tremble J, Morgenthaler NG, Vlug A et al. (1997) Human B cells secreting immunoglobulin $\mathrm{G}$ to glutamic acid decarboxylase-65 from a non-diabetic patient with multiple autoantibodies and Graves disease: a comparison with those present in Type I diabetes. J Clin Endocrinol Metab 82: 2664-2670

32. Powers AC, Bavik K, Tremble J, Daw K, Scherbaum WA, Banga JP (1999) Comparative analysis of epitope recognition of glutamic acid decarboxylase by autoantibodies from different autoimmune disorders. Clin Exp Immunol 118: 349-356

33. Schwartz H, Chandonia JM, Kash S et al. (1999) High-resolution autoreactive epitope mapping and structural modeling of the $65 \mathrm{kDa}$ form of human glutamic acid decarboxylase. J Mol Biol 287: 983-999

34. Maes M, Eisenbarth GS (1999) The polyglandular Autoimmune syndromes. In: Volpe R (ed) Autoimmune Endocrinopathies. Humana Press, New Jersey, pp 349-363

35. Schmidli RS, Colman PG, Bonifacio E (1995) Disease sensitivity and specificity of 52 assays for glutamic acid decarboxylase antibodies. The Second International GADAB Workshop. Diabetes 44: 636-640

36. Hatfield ECI, Hawkes CJ, Payton MA, Christie MR (1997) Cross reactivity between IA- 2 and phogrin/IA- $2 \beta$ in binding of autoantibodies in IDDM. Diabetologia 40: $1327-1333$ 
37. Moody AJ, Hejnaes KR, Marshall MO et al. (1995) Isolation by anion exchange of immunologically and enzymmatically active human islet glutamic acid decarboxylase 65 overexpressed in Sf9 insect cells. Diabetologia 38: 14-23

38. Li L, Jiang J, Hagopian WA et al. (1995) Differentail detection of rat islet and brain glutamic acid decarboxylase (GAD) isoforms with sequence-specific peptide antibodies. J Histochem Cytochem 43: 53-59

39. Christie MR, Brown TJ, Cassidy D (1992) Binding of antibodies in sera from Type I diabetic patients to glutamate decarboxylase from rat tissue. Evidence for antigenic and non-antigenic forms of the enzyme. Diabetologia 35: 380-384

40. Tindall KR, Kunkel TA (1988) Fidelity of DNA synthesis by the Thermus aquaticus DNA polymerase. Biochemistry 27: 6008-6013
41. Lee DS, Tian J, Phan T, Kaufman DL (1993) Cloning and sequence analysis of a murine cDNA encoding glutamate decarboxylase (GAD65). Biochim Biophys Acta 1216: 157-160

42. Suzuki R, Asami N, Amann E, Wagatsuma M (1995) Sequences of two porcine glutamic acid deacrboxylases (65and 67-kDa GAD). Gene 152: 257-260

43. Richter W, Northemann W, Muller M, Boehm BO (1996) Mapping of an autoreactive epitope with glutamate deacrboxylase using a diabetes-associated human monoclonal autoantibody and an epitope cDNA library. Hybridoma 15: 103-108

44. Bingley PJ, Christie MR, Bonifacio E et al. (1994) Combined analysis of autoantibodies improves prediction of IDDM in islet cell antibody-positive relatives. Diabetes 43: 1304-1310 